CRYSTALLOGRAPHIC COMMUNICATIONS

ISSN 2056-9890

Received 15 April 2019

Accepted 11 July 2019

Edited by E. V. Boldyreva, Russian Academy of Sciences, Russia

Keywords: crystal structure; tetrathiafulvalene; derivative; weak interactions; Hirshfeld surface analysis; DFT calculations.

CCDC reference: 1940080

Supporting information: this article has supporting information at journals.iucr.org/e

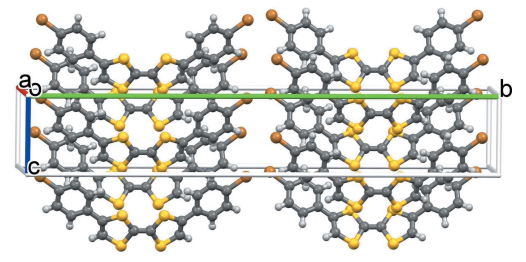

OPEN $\odot$ ACCESS

\section{Crystal structure of 4,4'-bis(4-bromophenyl)- $1,1^{\prime}, 3,3^{\prime}$-tetrathiafulvalene}

\author{
Sergei Rigin* and Marina Fonari
}

Department of Chemistry, New Mexico Highlands University, Las Vegas, New Mexico, 87701, USA. *Correspondence e-mail: rigindale@gmail.com

The molecule of the title compound, $\mathrm{C}_{18} \mathrm{H}_{10} \mathrm{Br}_{2} \mathrm{~S}_{4}$, has a $\mathrm{C}$-shape, with $C_{\mathrm{s}}$ molecular symmetry. The dihedral angle between the planes of the dithiol and phenyl rings is $8.35(9)^{\circ}$. In the crystal, molecules form helical chains along [001], the shortest interactions being $\pi \cdots S$ contacts within the helices. The intermolecular interactions were investigated by Hirshfeld surface analysis. Density functional theory (DFT) was used to calculate HOMO-LUMO energy levels of the title compound and its trans isomer.

\section{Chemical context}

So far significant progress has been achieved in improving the performance of organic field-effect transistors (OFETs) using such materials as oligoacenes, oligothiophenes and polythiophenes (Mas-Torrent \& Rovira, 2011; Pfattner, et al., 2016). Numerous derivatives of the sulfur heterocycle 2,2'-bis(1,3dithiolylidene), known as tetrathiafulvalene (TTF), have been noted as components of OFETs (Fourmigué \& Batail, 2004; Bendikov et al., 2004). High charge mobilities have been reported for thiophene-fused TTF and dibenzo-TTF in singlecrystal OFETs obtained from solutions, as well as in tetra(octadecylthio)-TTF films (Mas-Torrent et al., 2004a,b). A comparatively high mobility was reported for biphenylsubstituted TTF (Noda et al., 2005, 2007). Correlations between mobilities and herring-bone crystal structures have been investigated (Pfattner, et al., 2016; Mas-Torrent \& Rovira, 2011), including for phenyl-substituted oligothiophenes (Noda et al., 2007). Among the numerous reported halogenated tetrathiafulvalenes (Fourmigué \& Batail, 2004), only a few have been crystallographically characterized. The synthesis and characterization of two halogen TTF derivatives, 4,4'-bis(4-chlorophenyl)tetrathiafulvalene and 4,4'-bis(4bromophenyl)tetrathiafulvalene have been reported, but only the crystal structure of the chloro-substituted compound has been documented (Madhu \& Das, 2008), which shows short $\mathrm{Cl}$.. Cl contacts. Herein, we report the crystal structure, the Hirshfeld surface analysis and the molecular orbital analysis of the title compound, 4,4'-bis(4-bromophenyl)-1,1'3,3'-tetrathiafulvalene (BBP-TTF).<smiles>Brc1ccc(C2=CSC(=C3SC=C(c4ccc(Br)cc4)S3)S2)cc1</smiles> 


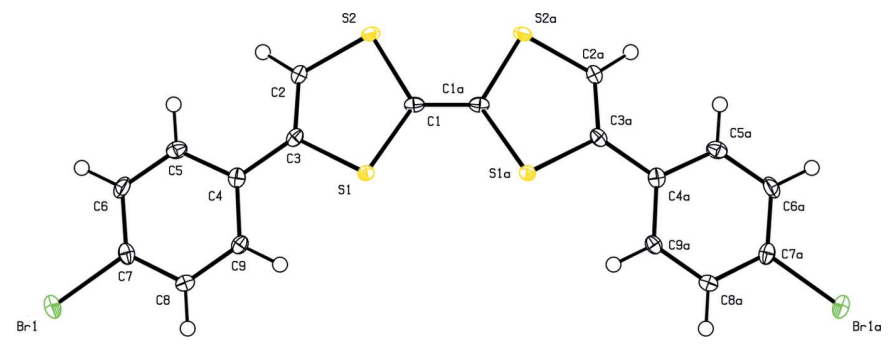

Figure 1

A view of the molecular structure of the title compound with the atom labelling. Displacement ellipsoids are drawn at the $50 \%$ probability level. Suffix a corresponds to the symmetry operation $x,-y+\frac{3}{2}, z$.

\section{Structural commentary}

The molecular structure of the title compound is illustrated in Fig. 1. The molecule has a C-shape with $C_{\mathrm{s}}$ molecular symmetry and resides on the mirror plane passing through the central $\mathrm{C} 1=\mathrm{C} 1(x,-\mathrm{y}+3 / 2, z)$ bond $[1.343(7) \AA]$. The $\mathrm{C}-\mathrm{S}$ distances in the TTF moiety are in the range 1.729 (4)1.778 (4) $\AA$ and correspond to reported values (CSD version 5.40, last update November 2018; Groom et al., 2016). The dihedral angle between the dithiol and phenyl rings is $8.35(9)^{\circ}$.

\section{Supramolecular features}

In the crystal (Fig. 2), no significant intermolecular interactions were found. Molecules related by the twofold screw axis form helices along the $c$-axis direction. The dihedral angle between the mean planes of the adjacent molecules in the helix is $36.59(3)^{\circ}$ and the helical pitch is 6.1991 (5) $\AA$. The shortest interactions within the chain, as indicated by Mercury (Macrae et al., 2006), are the $\mathrm{S} \cdots \pi$ contacts $\mathrm{C} 3 \cdots \mathrm{S} 2(1-x, y$, $\left.z-\frac{1}{2}\right)=3.458$ (4) and $\mathrm{C} 2 \cdots \mathrm{S} 2\left(1-x, y, z-\frac{1}{2}\right)=3.465$ (4) $\AA$, followed by the $\mathrm{C} 2-\mathrm{H} 2 \cdots \mathrm{C} 4\left(1-x, y, \frac{1}{2}+z\right)[2.72$, 3.467 (5) $\AA$ ] short contacts that are in agreement with the Hirshfeld (1977) surface analysis.

\section{Hirshfeld surface analysis}

CrystalExplorer17.5 (Wolff et al., 2012, Mackenzie et al., 2017) was used to generate the molecular Hirshfeld surface. The

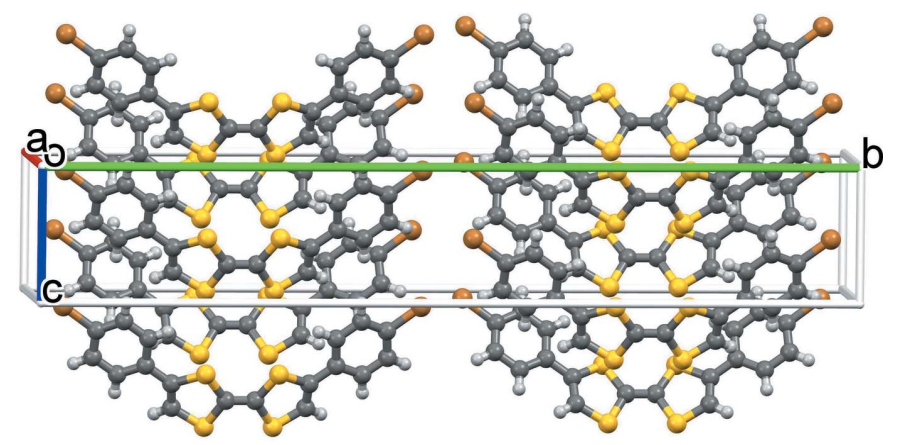

Figure 2

The crystal packing of the title compound.

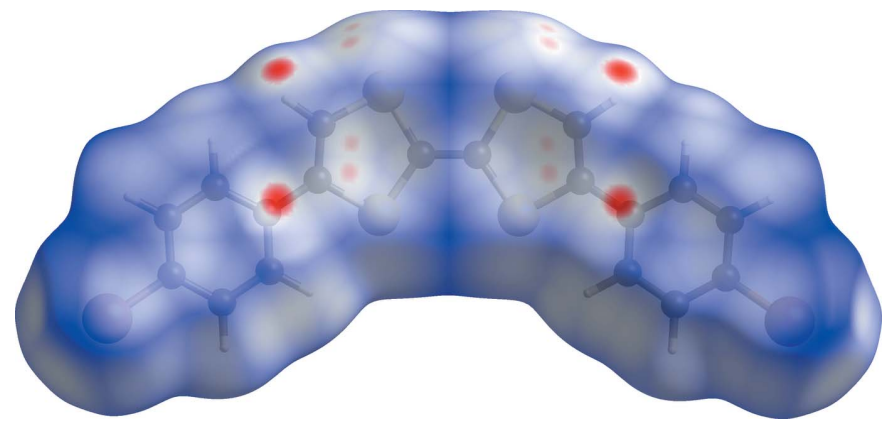

Figure 3

Hirshfeld surface mapped over $d_{\text {norm }}$ for the title compound in the range -0.1138 to 1.1257 a.u.

total $d_{\text {norm }}$ surface of the title compound is shown in Fig. 3 where the red spots correspond to the most significant interactions in the crystal. In the studied molecule, they include only weak $\mathrm{C}-\mathrm{H} \cdots \pi$ interactions at distances that are slightly higher than the sum of van der Waals radii.

\section{Frontier molecular orbital calculations}

The highest occupied molecular orbital (HOMO) acts as an electron donor and the lowest unoccupied molecular orbital

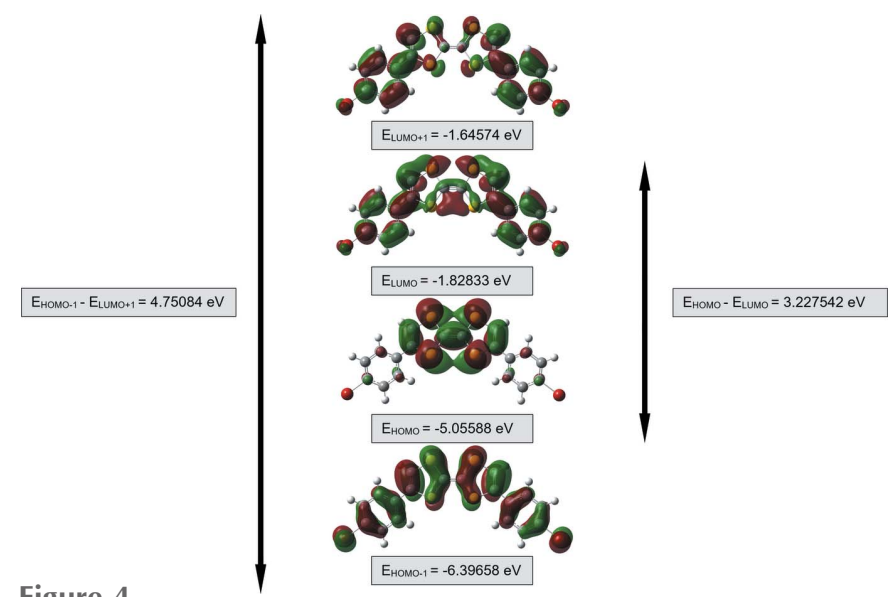

Figure 4

Molecular orbital energy levels of the title compound (cis isomer).

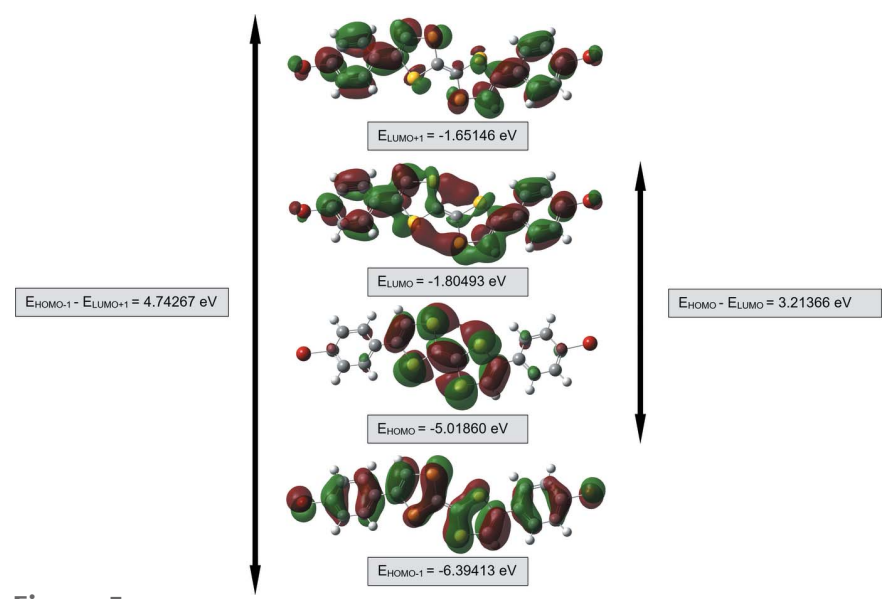

Figure 5

Molecular orbital energy levels of the trans isomer of the title compound. 
Table 1

Calculated frontier molecular orbital energies $(\mathrm{eV})$ for the title compound, its trans isomer and unsubstituted TTF and the conformational energy differences $\left(\mathrm{kJ} \mathrm{mol}^{-1}\right)$ between the cis and trans isomers.

\begin{tabular}{lrrr}
\hline & $c i s$ isomer & trans isomer & TTF \\
\hline$E$ (HOMO) & -5.0559 & -5.0186 & -4.8488 \\
$E($ LUMO) & -1.8283 & -1.8049 & -1.1252 \\
$E($ HOMO-1) & -6.3966 & -6.3941 & -6.6303 \\
$E$ (LUMO+1) & -1.6457 & -1.6515 & -0.7140 \\
$\Delta E($ HOMO-LUMO) & 3.2275 & 3.2137 & 3.7236 \\
$\Delta E$ (HOMO-1-LUMO+1) & 4.7508 & 4.7427 & 5.9163 \\
& & & \\
Chemical hardness $(\eta)$ & 1.6138 & 1.6068 & 1.8618 \\
Chemical potential $(\mu)$ & 3.4421 & 3.4118 & 2.9870 \\
Electronegativity $(\chi)$ & -3.4421 & -3.4118 & -2.9870 \\
Electrophilicity index $(\omega)$ & 3.6709 & 3.6221 & 2.3961 \\
& & & \\
$\Delta E($ cis-trans $)$ & 1.6331 & & \\
\hline
\end{tabular}

(LUMO) acts as an electron acceptor. A small HOMOLUMO energy gap indicates a highly polarizable molecule and high chemical reactivity. Molecular orbital energy levels for the title compound were calculated with Gaussian $16 \mathrm{~W}$ software (Frisch et al., 2016) using density functional theory (DFT) at the B3LYP/6-311+G(d,p) level of theory. The frontier orbitals of the title compound and its trans-isomer are shown in Figs. 4 and 5, respectively. The energy gap determines chemical hardness, chemical potential, electronegativity and the electrophilicity index. The orbital energy values for the title compound, its trans-isomer and unsubstituted TTF are summarized in Table 1. The conformation energy difference between the cis- and trans isomers is $1.6331 \mathrm{~kJ} \mathrm{~mol}^{-1}$. For both isomers the energy gap is large; hence both molecules are considered to be hard materials and would be difficult to polarize. As seen from Table 1, the bromophenyl substituents reduce the HOMO-LUMO energy gap and therefore the unsubstituted TTF molecule would be even more difficult to polarize.

\section{Database survey}

A search of the Cambridge Structural Database (CSD version 5.40, last update November 2018, Groom et al., 2016) for substituted TTF-phenyl derivatives related to the title compound yielded six structures. They include: bis $\left(4,4^{\prime}-\right.$ diphenyltetrathiafulvalenium)bis(pentafluorophenyl)gold(I) (CAKTAJ; Cerrada et al., 1998), 4,5'-diphenyltetrathiafulvalene (DPTFUL; Escande \& Lapasset, 1979, and DPTFUL01; Noda et al., 2007), 4,4'-bis(4-chlorophenyl)1,1',3,3'-tetrathiafulvalene (GOBVUP; Madhu \& Das, 2008), $4,5^{\prime}$-bis ( $p$-tolyl)tetrathiafulvalene (MOPJOR; Noda et al., 2007), 4,5'-bis(4-ethylphenyl)tetrathiafulvalene (MOPJUX; Noda et al., 2007), and 4,5'-bis(4-(trifluoromethyl)phenyl)tetrathiafulvalene (MOPKEI; Noda et al., 2007). Contrary to the title compound, they all exhibit inversion or pseudoinversion symmetry with a trans-arrangement of the phenyl substituents about the central $\mathrm{C}=\mathrm{C}$ bond. The $\mathrm{C}=\mathrm{C}$ bond lengths vary from $1.339 \AA$ (MOPJUX) to $1.353 \AA$ (DPTFUL);
Table 2

Experimental details.

\begin{tabular}{ll}
\hline Crystal data & \\
Chemical formula & $\mathrm{C}_{18} \mathrm{H}_{10} \mathrm{Br}_{2} \mathrm{~S}_{4}$ \\
$M_{\mathrm{r}}$ & 514.32 \\
Crystal system, space group & Orthorhombic, $A b m 2$ \\
Temperature $(\mathrm{K})$ & 90 \\
$a, b, c(\AA)$ & $7.5981(6), 37.411(3), 6.1991(5)$ \\
$V\left(\AA^{3}\right)$ & $1762.1(2)$ \\
$Z$ & 4 \\
Radiation type & Mo $K \alpha$ \\
$\mu\left(\mathrm{mm}^{-1}\right)$ & 5.07 \\
Crystal size (mm) & $0.17 \times 0.11 \times 0.05$ \\
& \\
Data collection & Bruker APEXII CCD \\
Diffractometer & Multi-scan $(S A D A B S ;$ Bruker, \\
Absorption correction & $2016)$ \\
& $0.625,0.747$ \\
$T_{\text {min }}, T_{\text {max }}$ & $34235,1580,1530$ \\
No. of measured, independent and & \\
$\quad$ observed $[I>2 \sigma(I)]$ reflections & 0.066 \\
$R_{\text {int }}$ & 0.594 \\
(sin $\theta / \lambda)_{\text {max }}\left(\AA^{-1}\right)$ & \\
Refinement & \\
$R\left[F^{2}>2 \sigma\left(F^{2}\right)\right], w R\left(F^{2}\right), S$ & $0.017,0.041,1.09$ \\
No. of reflections & 1580 \\
No. of parameters & 109 \\
No. of restraints & 1 \\
H-atom treatment & H-atom parameters constrained \\
$\Delta \rho_{\text {max }}, \Delta \rho_{\min }\left(\mathrm{e} \AA^{-3}\right)$ & $0.29,-0.29$ \\
Absolute structure & Flack $x$ determined using 663 \\
& quotients $\left[\left(I^{+}\right)-\left(I^{-}\right)\right] /\left[\left(I^{+}\right)+\left(I^{-}\right)\right]$ \\
Absolute structure parameter & $0.014(5)$ \\
\hline
\end{tabular}

Computer programs: APEX2 and SAINT (Bruker, 2016), SHELXT (Sheldrick, 2015a), SHELXL2018 (Sheldrick, 2015b) and OLEX2 (Dolomanov et al., 2009).

the value observed for the title compound falls within this limit. All of the above molecules are almost planar, with tilt angles between the dithiol and phenyl rings varying from 5.39 to $10.18^{\circ}$ for the two independent molecules in DPTFUL01 to $28.28^{\circ}$ in GOBVUP and $30.29^{\circ}$ in MOPKEI; the greatest twisting was observed for halogen-substituted derivatives.

\section{Crystallization}

The single crystals of the title compound were obtained in attempt to co-crystallize it with tetracyanoquinodimethane (TCNQ) in a 1:1 molar ratio. A saturated solution of 4,4'-bis(4bromophenyl)-1,1'3,3'-tetrathiafulvalene (2 mg, Aldrich) in chloroform was mixed with a saturated solution of TCNQ ( $1 \mathrm{mg}$, Aldrich) in acetonitrile and left at room temperature. Red prismatic crystals suitable for the X-ray diffraction analysis were obtained after a week of slow evaporation.

\section{Refinement}

Crystal data, data collection and structure refinement details are summarized in Table 2. The hydrogen atoms were positioned geometrically and refined using a riding model: $\mathrm{C}-\mathrm{H}=$ $0.93 \AA$ with $U_{\text {iso }}(\mathrm{H})=1.2 U_{\text {eq }}(\mathrm{C})$. 


\section{Funding information}

Funding for this research was provided by: NSF DMR 1523611 (PREM).

\section{References}

Bendikov, M., Wudl, F. \& Perepichka, D. F. (2004). Chem. Rev. 104, 4891-4946.

Bruker (2016). SAINT, APEX2 and SADABS. Bruker AXS Inc., Madison, Wisconsin, USA.

Cerrada, E., Laguna, M., Bartolomé, J., Campo, J., Orera, V. \& Jones, P. G. (1998). Synth. Met. 92, 245-251.

Dolomanov, O. V., Bourhis, L. J., Gildea, R. J., Howard, J. A. K. \& Puschmann, H. (2009). J. Appl. Cryst. 42, 339-341.

Escande, A. \& Lapasset, J. (1979). Cryst. Struct. Commun. 8, 1009. Fourmigué, M. \& Batail, P. (2004). Chem. Rev. 104, 5379-5418.

Frisch, M. J., Trucks, G. W., Schlegel, H. B., Scuseria, G. E., Robb, M. A., Cheeseman, J. R., Scalmani, G., Barone, V., Petersson, G. A., Nakatsuji, H., Li, X., Caricato, M., Marenich, A. V., Bloino, J., Janesko, B. G., Gomperts, R., Mennucci, B., Hratchian, H. P., Ortiz, J. V., Izmaylov, A. F., Sonnenberg, J. L., Williams-Young, D., Ding, F., Lipparini, F., Egidi, F., Goings, J., Peng, B., Petrone, A., Henderson, T., Ranasinghe, D., Zakrzewski, V. G., Gao, J., Rega, N., Zheng, G., Liang, W., Hada, M., Ehara, M., Toyota, K., Fukuda, R., Hasegawa, J., Ishida, M., Nakajima, T., Honda, Y., Kitao, O., Nakai, H., Vreven, T., Throssell, K., Montgomery, J. A., Jr, Peralta, J. E., Ogliaro, F., Bearpark, M. J., Heyd, J. J., Brothers, E. N., Kudin, K. N., Staroverov, V. N., Keith, T. A., Kobayashi, R., Normand, J, Raghavachari, K.,; Rendell, A. P., Burant, J. C., Iyengar, S. S., Tomasi, J., Cossi, M., Millam, J. M., Klene, M., Adamo, C., Cammi, R., Ochterski, J. W., Martin, R. L., Morokuma, K., Farkas, O.,
Foresman, J. B. \& Fox, D. J. (2016). Gaussian 16W. Gaussian, Inc., Wallingford CT, USA.

Groom, C. R., Bruno, I. J., Lightfoot, M. P. \& Ward, S. C. (2016). Acta Cryst. B72, 171-179.

Hirshfeld, F. L. (1977). Theor. Chim. Acta, 44, 129-138.

Mackenzie, C. F., Spackman, P. R., Jayatilaka, D. \& Spackman, M. A. (2017). IUCrJ, 4, 575-587.

Macrae, C. F., Edgington, P. R., McCabe, P., Pidcock, E., Shields, G. P., Taylor, R., Towler, M. \& van de Streek, J. (2006). J. Appl. Cryst. 39, 453-457.

Madhu, V. \& Das, S. K. (2008). Inorg. Chem. 47, 5055-5070.

Mas-Torrent, M., Durkut, M., Hadley, P., Ribas, X. \& Rovira, C. (2004a). J. Am. Chem. Soc. 126, 984-985.

Mas-Torrent, M., Hadley, P., Bromley, S. T., Ribas, X., Tarrés, J., Mas, M., Molins, E., Veciana, J. \& Rovira, C. (2004b). J. Am. Chem. Soc. 126, 8546-8553.

Mas-Torrent, M. \& Rovira, C. (2011). Chem. Rev. 111, 4833-4856.

Noda, B., Katsuhara, M., Aoyagi, I., Mori, T., Taguchi, T., Kambayashi, T., Ishikawa, K. \& Takezoe, H. (2005). Chem. Lett. 34, 392-393.

Noda, B., Wada, H., Shibata, K., Yoshino, T., Katsuhara, M., Aoyagi, I., Mori, T., Taguchi, T., Kambayashi, T., Ishikawa, K. \& Takezoe, H. (2007). Nanotechnology, 18, 424009.

Parsons, S., Flack, H. D. \& Wagner, T. (2013). Acta Cryst. B69, 249 259.

Pfattner, R., Bromley, S. T., Rovira, C. \& Mas-Torrent, M. (2016). Adv. Funct. Mater. 26, 2256-2275.

Sheldrick, G. M. (2015a). Acta Cryst. A71, 3-8.

Sheldrick, G. M. (2015b). Acta Cryst. C71, 3-8.

Wolff, S. K., Grimwood, D. J., McKinnon, J. J., Turner, M. J., Jayatilaka, D. \& Spackman, M. A. (2012). CrystalExplorer. University of Western Australia, Australia. 


\section{supporting information}

Acta Cryst. (2019). E75, 1195-1198 [https://doi.org/10.1107/S2056989019009952]

\section{Crystal structure of 4,4'-bis(4-bromophenyl)-1,1',3,3'-tetrathiafulvalene}

\section{Sergei Rigin and Marina Fonari}

\section{Computing details}

Data collection: APEX2 (Bruker, 2016); cell refinement: SAINT (Bruker, 2016); data reduction: SAINT (Bruker, 2016); program(s) used to solve structure: SHELXT (Sheldrick, 2015a); program(s) used to refine structure: SHELXL2018 (Sheldrick, 2015b); molecular graphics: OLEX2 (Dolomanov et al., 2009); software used to prepare material for publication: OLEX2 (Dolomanov et al., 2009).

4,4'-Bis(4-bromophenyl)-1, 1',3,3'-tetrathiafulvalene

Crystal data

$\mathrm{C}_{18} \mathrm{H}_{10} \mathrm{Br}_{2} \mathrm{~S}_{4}$

$M_{r}=514.32$

Orthorhombic, $A b m 2$

$a=7.5981$ (6) $\AA$

$b=37.411(3) \AA$

$c=6.1991(5) \AA$

$V=1762.1(2) \AA^{3}$

$Z=4$

$F(000)=1008$

\section{Data collection}

Bruker APEXII CCD diffractometer

$\varphi$ and $\omega$ scans

Absorption correction: multi-scan

(SADABS; Bruker, 2016)

$T_{\min }=0.625, T_{\max }=0.747$

34235 measured reflections

\section{Refinement}

Refinement on $F^{2}$

Least-squares matrix: full

$R\left[F^{2}>2 \sigma\left(F^{2}\right)\right]=0.017$

$w R\left(F^{2}\right)=0.041$

$S=1.09$

1580 reflections

109 parameters

1 restraint

Primary atom site location: dual

Hydrogen site location: inferred from neighbouring sites
$D_{\mathrm{x}}=1.939 \mathrm{Mg} \mathrm{m}^{-3}$

Mo $K \alpha$ radiation, $\lambda=0.71073 \AA$

Cell parameters from 9390 reflections

$\theta=2.2-28.4^{\circ}$

$\mu=5.07 \mathrm{~mm}^{-1}$

$T=90 \mathrm{~K}$

Prism, red

$0.17 \times 0.11 \times 0.05 \mathrm{~mm}$

1580 independent reflections 1530 reflections with $I>2 \sigma(I)$

$R_{\text {int }}=0.066$

$\theta_{\text {max }}=25.0^{\circ}, \theta_{\min }=1.1^{\circ}$

$h=-9 \rightarrow 9$

$k=-44 \rightarrow 44$

$l=-7 \rightarrow 7$

H-atom parameters constrained

$w=1 /\left[\sigma^{2}\left(F_{\mathrm{o}}^{2}\right)+(0.0126 P)^{2}+2.1911 P\right]$ where $P=\left(F_{\mathrm{o}}{ }^{2}+2 F_{\mathrm{c}}{ }^{2}\right) / 3$

$(\Delta / \sigma)_{\max }=0.003$

$\Delta \rho_{\max }=0.29 \mathrm{e} \AA^{-3}$

$\Delta \rho_{\min }=-0.29$ e $\AA^{-3}$

Absolute structure: Flack $x$ determined using 663 quotients $\left[\left(I^{+}\right)-(I)\right] /\left[\left(I^{+}\right)+(I)\right]$ (Parsons et al., 2013)

Absolute structure parameter: 0.014 (5) 


\section{Special details}

Geometry. All esds (except the esd in the dihedral angle between two 1.s. planes) are estimated using the full covariance matrix. The cell esds are taken into account individually in the estimation of esds in distances, angles and torsion angles; correlations between esds in cell parameters are only used when they are defined by crystal symmetry. An approximate (isotropic) treatment of cell esds is used for estimating esds involving l.s. planes.

Fractional atomic coordinates and isotropic or equivalent isotropic displacement parameters $\left(\AA^{2}\right)$

\begin{tabular}{lllll}
\hline & $x$ & $y$ & $z$ & $U_{\text {iso }} * / U_{\text {eq }}$ \\
\hline Br1 & $0.79020(5)$ & $0.53149(2)$ & $0.04905(9)$ & $0.02338(12)$ \\
S1 & $0.84814(9)$ & $0.70527(2)$ & $0.54322(17)$ & $0.01212(17)$ \\
S2 & $0.64506(12)$ & $0.70791(2)$ & $0.95068(14)$ & $0.01322(19)$ \\
C1 & $0.7514(4)$ & $0.73205(10)$ & $0.7447(6)$ & $0.0120(7)$ \\
C2 & $0.6459(5)$ & $0.66817(10)$ & $0.8081(6)$ & $0.0122(8)$ \\
H2 & 0.583254 & 0.648059 & 0.861560 & $0.015^{*}$ \\
C3 & $0.7350(5)$ & $0.66579(9)$ & $0.6225(6)$ & $0.0117(8)$ \\
C4 & $0.7515(4)$ & $0.63362(9)$ & $0.4874(6)$ & $0.0125(8)$ \\
C5 & $0.6886(4)$ & $0.60030(9)$ & $0.5600(9)$ & $0.0159(7)$ \\
H5 & 0.637106 & 0.598599 & 0.699223 & $0.019^{*}$ \\
C6 & $0.7004(5)$ & $0.56997(10)$ & $0.4326(7)$ & $0.0182(8)$ \\
H6 & 0.658485 & 0.547656 & 0.484660 & $0.022^{*}$ \\
C7 & $0.7737(5)$ & $0.57250(10)$ & $0.2289(7)$ & $0.0148(8)$ \\
C8 & $0.8384(4)$ & $0.60494(10)$ & $0.1519(6)$ & $0.0133(7)$ \\
H8 & 0.890749 & 0.606365 & 0.012995 & $0.016^{*}$ \\
C9 & $0.8253(4)$ & $0.63529(10)$ & $0.2817(6)$ & $0.0129(8)$ \\
H9 & 0.867405 & 0.657541 & 0.228922 & $0.015^{*}$ \\
& & & &
\end{tabular}

Atomic displacement parameters $\left(\AA^{2}\right)$

\begin{tabular}{lllllll}
\hline & $U^{11}$ & $U^{22}$ & $U^{33}$ & $U^{12}$ & $U^{13}$ & $U^{23}$ \\
\hline Br1 & $0.0322(2)$ & $0.01276(17)$ & $0.02519(19)$ & $-0.00010(14)$ & $0.0039(2)$ & $-0.0046(2)$ \\
S1 & $0.0126(4)$ & $0.0117(4)$ & $0.0120(4)$ & $-0.0005(3)$ & $0.0032(5)$ & $0.0006(5)$ \\
S2 & $0.0148(4)$ & $0.0149(4)$ & $0.0100(4)$ & $0.0001(4)$ & $0.0030(4)$ & $0.0018(4)$ \\
C1 & $0.0071(15)$ & $0.0181(17)$ & $0.0109(16)$ & $0.0014(14)$ & $0.0010(12)$ & $0.0008(15)$ \\
C2 & $0.0111(17)$ & $0.0111(19)$ & $0.0145(18)$ & $-0.0007(13)$ & $-0.0011(14)$ & $0.0022(14)$ \\
C3 & $0.0089(17)$ & $0.0126(19)$ & $0.0134(18)$ & $0.0024(13)$ & $-0.0025(12)$ & $0.0042(13)$ \\
C4 & $0.0074(15)$ & $0.0130(18)$ & $0.017(2)$ & $0.0019(12)$ & $-0.0022(12)$ & $0.0010(13)$ \\
C5 & $0.0136(15)$ & $0.0191(17)$ & $0.0149(16)$ & $0.0002(12)$ & $0.0022(19)$ & $0.002(2)$ \\
C6 & $0.022(2)$ & $0.0124(19)$ & $0.020(2)$ & $0.0007(15)$ & $0.0009(16)$ & $0.0067(17)$ \\
C7 & $0.0141(18)$ & $0.0119(19)$ & $0.0186(19)$ & $0.0014(14)$ & $-0.0036(16)$ & $-0.0017(16)$ \\
C8 & $0.0121(18)$ & $0.0160(19)$ & $0.0117(17)$ & $-0.0007(14)$ & $0.0003(15)$ & $0.0017(15)$ \\
C9 & $0.0115(17)$ & $0.0127(19)$ & $0.0144(18)$ & $0.0007(14)$ & $-0.0014(14)$ & $0.0029(15)$ \\
& & & & & &
\end{tabular}

Geometric parameters $\left(\AA,{ }^{o}\right)$

\begin{tabular}{llll}
\hline $\mathrm{Br} 1-\mathrm{C} 7$ & $1.901(4)$ & $\mathrm{C} 4-\mathrm{C} 9$ & $1.394(5)$ \\
$\mathrm{S} 1-\mathrm{C} 1$ & $1.762(4)$ & $\mathrm{C} 5-\mathrm{H} 5$ & 0.9500 \\
$\mathrm{~S} 1-\mathrm{C} 3$ & $1.778(4)$ & $\mathrm{C} 5-\mathrm{C} 6$ & $1.385(6)$
\end{tabular}




\begin{tabular}{|c|c|c|c|}
\hline $\mathrm{S} 2-\mathrm{C} 1$ & $1.760(4)$ & $\mathrm{C} 6-\mathrm{H} 6$ & 0.9500 \\
\hline $\mathrm{S} 2-\mathrm{C} 2$ & $1.729(4)$ & $\mathrm{C} 6-\mathrm{C} 7$ & $1.384(6)$ \\
\hline $\mathrm{C} 1-\mathrm{C}^{\mathrm{i}}$ & $1.343(7)$ & $\mathrm{C} 7-\mathrm{C} 8$ & $1.394(5)$ \\
\hline $\mathrm{C} 2-\mathrm{H} 2$ & 0.9500 & $\mathrm{C} 8-\mathrm{H} 8$ & 0.9500 \\
\hline $\mathrm{C} 2-\mathrm{C} 3$ & $1.338(5)$ & $\mathrm{C} 8-\mathrm{C} 9$ & $1.396(6)$ \\
\hline $\mathrm{C} 3-\mathrm{C} 4$ & $1.472(5)$ & $\mathrm{C} 9-\mathrm{H} 9$ & 0.9500 \\
\hline $\mathrm{C} 4-\mathrm{C} 5$ & $1.409(5)$ & & \\
\hline $\mathrm{C} 1-\mathrm{S} 1-\mathrm{C} 3$ & $94.28(17)$ & $\mathrm{C} 6-\mathrm{C} 5-\mathrm{C} 4$ & $121.4(4)$ \\
\hline $\mathrm{C} 2-\mathrm{S} 2-\mathrm{C} 1$ & $93.94(18)$ & $\mathrm{C} 6-\mathrm{C} 5-\mathrm{H} 5$ & 119.3 \\
\hline $\mathrm{S} 2-\mathrm{C} 1-\mathrm{S} 1$ & $114.5(2)$ & $\mathrm{C} 5-\mathrm{C} 6-\mathrm{H} 6$ & 120.3 \\
\hline $\mathrm{C} 1-\mathrm{C} 1-\mathrm{S} 1$ & $124.66(13)$ & $\mathrm{C} 7-\mathrm{C} 6-\mathrm{C} 5$ & $119.4(4)$ \\
\hline $\mathrm{C} 1-\mathrm{C} 1-\mathrm{S} 2$ & $120.87(12)$ & $\mathrm{C} 7-\mathrm{C} 6-\mathrm{H} 6$ & 120.3 \\
\hline $\mathrm{S} 2-\mathrm{C} 2-\mathrm{H} 2$ & 120.1 & $\mathrm{C} 6-\mathrm{C} 7-\mathrm{Br} 1$ & $120.5(3)$ \\
\hline $\mathrm{C} 3-\mathrm{C} 2-\mathrm{S} 2$ & $119.9(3)$ & $\mathrm{C} 6-\mathrm{C} 7-\mathrm{C} 8$ & $120.9(4)$ \\
\hline $\mathrm{C} 3-\mathrm{C} 2-\mathrm{H} 2$ & 120.1 & $\mathrm{C} 8-\mathrm{C} 7-\mathrm{Br} 1$ & $118.6(3)$ \\
\hline $\mathrm{C} 2-\mathrm{C} 3-\mathrm{S} 1$ & $115.3(3)$ & $\mathrm{C} 7-\mathrm{C} 8-\mathrm{H} 8$ & 120.5 \\
\hline $\mathrm{C} 2-\mathrm{C} 3-\mathrm{C} 4$ & $125.9(3)$ & $\mathrm{C} 7-\mathrm{C} 8-\mathrm{C} 9$ & $119.1(3)$ \\
\hline $\mathrm{C} 4-\mathrm{C} 3-\mathrm{S} 1$ & $118.7(2)$ & $\mathrm{C} 9-\mathrm{C} 8-\mathrm{H} 8$ & 120.5 \\
\hline $\mathrm{C} 5-\mathrm{C} 4-\mathrm{C} 3$ & $120.9(3)$ & $\mathrm{C} 4-\mathrm{C} 9-\mathrm{C} 8$ & $121.3(3)$ \\
\hline $\mathrm{C} 9-\mathrm{C} 4-\mathrm{C} 3$ & $121.2(3)$ & $\mathrm{C} 4-\mathrm{C} 9-\mathrm{H} 9$ & 119.3 \\
\hline $\mathrm{C} 9-\mathrm{C} 4-\mathrm{C} 5$ & $117.9(4)$ & $\mathrm{C} 8-\mathrm{C} 9-\mathrm{H} 9$ & 119.3 \\
\hline $\mathrm{C} 4-\mathrm{C} 5-\mathrm{H} 5$ & 119.3 & & \\
\hline
\end{tabular}

Symmetry code: (i) $x,-y+3 / 2, z$. 